RUNNING HEAD: Losartan and emotion processing in the brain

Word count manuscript: 3,969; word count abstract: 237

2 Tables; 3 Figures; 3 Supplementary Tables

\title{
Angiotensin regulation of amygdala response to threat in high-trait anxious individuals
}

Andrea Reinecke ${ }^{1}$, Michael Browning ${ }^{1,2}$, Joppe Klein Breteler ${ }^{3}$, Nils Kappelmann ${ }^{1}$, Kerry J. Ressler $^{4}$, Catherine J. Harmer ${ }^{1}$, Michelle G. Craske ${ }^{5}$

${ }^{1}$ Department of Psychiatry, University of Oxford, UK

${ }^{2}$ Oxford Health NHS Trust, Oxford, UK

${ }^{3}$ Behavioural Science Institute, Radboud University Nijmegen, The Netherlands

${ }^{4}$ McLean Hospital, Harvard University, US

${ }^{5}$ Department of Psychology, University of California, Los Angeles, US

Corresponding author: Andrea Reinecke, University of Oxford, Department of Psychiatry, Warneford Hospital, Oxford OX37JX, UK, e-mail: andrea.reinecke@psych.ox.ac.uk, phone: +441865 618320, fax: +441865251076. 


\begin{abstract}
Background. The antihypertensive drug losartan has been shown to improve memory in humans as well as learning and fear extinction in rodent models, highlighting its potential to have similar synergistic effects on exposure-based cognitive-behavior therapy (CBT) for anxiety disorders. This study investigated the effect of losartan on neural correlates of processing threat versus safety stimuli in highly anxious individuals, to identify potential pathways of how the drug might facilitate psychological treatment.
\end{abstract}

Methods. 30 healthy volunteers high in trait anxiety were randomly assigned to a single dose of losartan (50mg) versus placebo, before undergoing functional magnetic resonance imaging. We measured brain response to happy and fearful faces presented for 80 s, to assess emotional processing and habituation over time.

Results. The placebo group showed similarly high left amygdala activation early on during presentation of fearful and happy faces, which decreased over time. In contrast, losartan reduced amygdala response to happy faces early on. In response to fearful faces, the drug prevented habituation, caused sustained amygdala activation, and led to increased activation in other brain areas associated with threat processing, such as the insula and putamen. Conclusion. Our findings suggest two distinct effects of losartan on emotional processing, including an improvement of early discrimination of stimuli as threatening versus safe, and facilitation of threat processing. Both these processes are known to be relevant for successful exposure, highlighting two potential pathways by which losartan might exert facilitative effects on psychological treatment. (The Effect of Losartan on Emotional Processing in Healthy Volunteers; clinicaltrials.gov; NCT03434054)

keywords: amygdala, anxiety, emotional processing, fear extinction, habituation, losartan 


\section{Introduction}

There has been strong interest in the combination of psychological and pharmacological treatments for anxiety disorders to increase treatment efficacy and efficiency. Recent work shows that drugs targeting glutamatergic mechanisms, such as dcycloserine, can significantly improve the number of responders to exposure-based cognitivebehaviour therapy (1-3). However, an increasing number of studies also report no or even detrimental effects of d-cycloserine on clinical outcome (4). Such findings might be due to the drug not only enhancing fear extinction during successful exposure but also fear reconsolidation during unsuccessful sessions, leaving the risk of patients deteriorating (5).

A promising alternative add-on treatment to exposure-based therapies might be compounds targeting the renin-angiotensin system, which is predominantly involved in cardiovascular regulation. Angiotensin receptor antagonists such as losartan are routinely used to treat hypertension, but increasing evidence suggests that such drugs may also enhance cognition (6-10) and prevent the development of anxiety disorders in humans (11). Most importantly, recent studies suggest that angiotensin regulation may directly affect fear extinction in animal models $(12,13)$. A single dose of losartan prior to fear extinction in rodents - often thought of as an analogue to human exposure therapy - has been shown to significantly reduce fear response at retest, while having no effect on fear acquisition (14).

Drugs such as losartan block $\mathrm{AT}_{1}$ receptors, which leads to increased availability of angiotensin II-converted angiotensin IV, thought to play a crucial role in neuroplastic processes (15). Recent data suggest that angiotensin receptors are expressed within the brain as well as the periphery and can be found in brain regions involved in threat processing and fear conditioning, such as the amygdala, hippocampus and PFC (16-19). One recent study demonstrated that angiotensin receptors within the amygdala may be in part necessary for fear and threat processing (20). Such findings support the hypothesis that the angiotensin pathway may directly affect central nervous system threat processing in addition to peripheral 
regulatory pathways. Overall, these findings not only suggest that losartan might be an effective adjacent treatment to exposure to improve overall efficacy, but also that - different from d-cycloserine - it might have the potential to minimize drug-induced worsening of clinical symptoms after unsuccessful exposure.

This is the first study to investigate the underlying brain mechanisms by which losartan might affect emotional processing in humans, to explore possible synergies with exposure therapy for anxiety disorders. In a group of high-trait anxious individuals, we tested the effect of a single drug versus placebo administration on brain activation during prolonged exposure to fearful and happy facial expressions. Contemporary models of exposure therapy for anxiety disorders propose that an essential mechanism of treatment action is inhibitory learning (21). Through extended exposure to feared stimuli without the anticipated aversive consequences, stimuli previously perceived as predictive of threat are no longer predictive of threat, leading to an overwriting of stimulus-threat associations in memory. For successful inhibitory learning to take place during exposure, it is thought to be essential for attentional and emotional processing to be focused on the threat stimulus for a prolonged period of time, to allow prediction error correction (21-23). We therefore hypothesized that losartan would promote sustained threat processing in limbic areas in response to fearful faces and interrupt the typical pattern of habituation $(24,25)$.

\section{Methods and Materials}

\section{Participants}

Thirty high-trait anxious, healthy participants were recruited through local advertisements. Formal sample size calculation was limited by the lack of previous evidence regarding the effect of losartan on neuroimaging outcomes. We estimated sample size based on the only available study into the effect of losartan on memory in healthy volunteers. With 
observed prospective memory detection rates of $\mathrm{M}=3.9 / \mathrm{SD}=2.4$ after placebo and $\mathrm{M}=5.7 / \mathrm{SD}=1.6$ after losartan (7), conservative calculations suggested 15 participants per group to achieve effect sizes $d>0.8$ and a statistical power of $80 \%$ ( $\alpha$-level 0.05 ).

Participants were included in the study if they presented with a score of 40 or higher on the trait form of the State-Trait Anxiety Inventory (STAIT), to capture a sample scoring above published population means (26). All but 3 participants had scores of at least 45, resulting in high-trait anxiety samples similar to or more anxious than those tested in previous studies $(27,28)$. Participants were excluded if they met criteria for a current DSM-IV Axis I disorder as assessed using the Structured Clinical Interview for DSM-IV (29). Participants also had to be medication-free for at least 6 weeks, have a body mass index of $18-30 \mathrm{~kg} / \mathrm{m}^{2}$, be right-handed, have no contraindication to magnet resonance imaging (MRI), and have no first-degree family member with a history of a severe psychiatric disease. The study was approved by the Oxford University research ethics committee, and all participants gave written informed consent.

\section{Materials and Study Design}

To characterise the sample, all participants completed a questionnaire battery at screening: the Anxiety Sensitivity Index Revised (ASI-R) (30), the neuroticism subscale of the Eysenck Personality Inventory (EPI) (31); the Behavioural Inhibition Scale (BIS) (32), the Beck Depression Inventory (BDI) (33), and the Attentional Control Scale (ACS) (34). The National Adult Reading Test (NART) (35) was applied to estimate verbal intelligence. On the test day, participants were stratified for gender and randomly allocated to one of two treatment conditions in a double-blind design: losartan (Cozaar, Merck Sharp \& Dohme Ltd.) given as a single oral 50mg dose or matched placebo (microcrystalline cellulose; Rayotabs, Rayonex GmbH). Scanning began 1.5 hours after medication administration, when drug peak plasma levels are typically reached $(36,37)$. A sub-clinical dose of 50mg was chosen to be 
able to establish effects on emotional processing without potential confounding hypotensive effects (7).

To assess potential changes in subjective state mood and physiological symptoms, participants completed visual analogue scales before administration of medication and before testing, at which time we also measured heart rate and blood pressure, using an Omron 705IT sphygmomanometer.

\section{fMRI Image Acquisition and Analysis}

Task Design. Stimuli were black-and-white photographs of 4 female and 4 male faces, each once with a fearful and once with a happy expression (38). Experimental design and timings were based on previously published work using the same task (24, 25): Each participant was presented with four face-blocks of 80s each, each embedded between 20s lowlevel baseline fixation blocks. In each of these blocks, the same facial image was presented 160 times for 300ms, separated by 200ms interstimulus intervals. Each participant was presented two different female and two different male faces, with each gender showing one happy and one fearful facial expression. In each experiment, the two happy and two fearful blocks alternated, and 25\% of participants in each drug group started with a female happy, female fearful, male happy, versus male fearful block. Before each face block, participants were instructed to attend to the eye level of the face and remain alert. Immediately after the scan, participants were asked to identify the four faces seen during the scan presented among 12 distractor faces, and they gave valence (from -3 negative to +3 positive) and arousal (from 0 low to 6 high) ratings for each face (Figure 1).

Image Acquisition. Images were recorded using a 3T Siemens Trio scanner. 3D highresolution T1-weighted images were acquired for subject alignment, using an MPRAGE sequence with the following parameters: $174 \times 192 \times 192$ field of view matrix, flip angle $=8^{\circ}$, voxel resolution $1 \mathrm{~mm}^{3}$, TR=2040ms, TE=4.7ms, inversion time $(\mathrm{TI})=900 \mathrm{~ms}$, acquisition 
time $=5$ min $56 \mathrm{sec}$. $\mathrm{T}_{2}{ }^{*}$-weighted functional data were acquired for a whole-brain field-ofview using a gradient echo EPI sequence (147x192x192 field of view matrix, flip angle=87 voxel resolution $3 \mathrm{~mm}^{3}$, repetition time $(\mathrm{TR})=3000 \mathrm{~ms}$, echo time $(\mathrm{TE})=30 \mathrm{~ms}$, acquisition time $=7 \mathrm{~min} 36 \mathrm{sec}$ ). Field maps were acquired using a dual echo 2D gradient echo sequence with echos at 5.19 and $7.65 \mathrm{~ms}$, and a repetition time of $488 \mathrm{~ms}$.

Image Analysis. Imaging data were analysed using FSL (FMRIB Software Library; www.fmrib.ox.ac.ul/fsl). Registration to high resolution structural and/or standard space images was carried out using FLIRT (39). Registration from high resolution structural to standard space was then further refined using FNIRT nonlinear registration. Z statistic fMRI images were thresholded using clusters determined by $\mathrm{Z}>2.3$ and a (corrected) cluster significance threshold of $\mathrm{P}=0.05$. Pre-processing included motion correction using MCFLIRT (39), non-brain removal using BET (40), slice-timing correction using Fourier-space timeseries phase-shifting, fieldmap correction, spatial smoothing using a Gaussian kernel of FWHM 6.0mm, grand-mean intensity normalisation of the entire 4D dataset by a single multiplicative factor, highpass temporal filtering (Gaussian-weighted least-squares straight line fitting, with sigma=45.0s).

At the first-level, fMRI data were analysed using a general linear model approach with local autocorrelation correction (41). Four regressors of interest were modelled, including the first (early) and last (late) 40s of fearful versus happy facial expression blocks. Fixation blocks were the implicit baseline reference. The main contrasts of interests to assess response to valence were: fear versus happy blocks (and vice versa) over the whole 80s presentation, fear versus happy (and vice versa) for early presentation (first 40s per block), and fear versus happy (and vice versa) for late presentation (last 40s). The main contrasts of interest to assess response habituation were: early (first 40s per block) versus late (last 40s) presentation (and vice versa) collapsed across fearful and happy faces, early versus late presentation (and vice versa) for fearful facial expressions, and early versus late presentation (and vice versa) for 
happy facial expressions. Individual activation maps were then entered into the group level, using a mixed-effects whole-brain analysis (42).

Based on previous work identifying the bilateral amygdala as relevant in response habituation using this task $(24,25)$ we also ran region of interest analyses (ROI), including anatomical masks of both amygdala. Significant whole-brain and ROI interactions were explored by entering extracted percent blood oxygenation level-dependent (BOLD) signal changes valence (fear, happy) x time (early, late) x group ANOVAs.

\section{Statistical Analysis of Non-imaging Variables}

Statistical analyses of non-imaging variables were carried out using SPSS 20 software (SPSS, Inc., Chicago IL), using two-tailed tests and $a=0.05$. Potential drug-induced changes in mood and physiological parameters were assessed using independent-samples t-tests on peak-level scores. Group differences in recognition rate, arousal and valence ratings for experimental faces were assessed using independent-samples t-tests.

\section{Results}

\section{Group Matching, Drug Side Effects, and Facial Stimuli}

The groups were well-matched on sociodemographic, clinical and personality parameters. There were no group differences in peak-level blood pressure and heart rate, state mood and physiological symptoms. Participants in the two groups were also no different with regards to their valence and arousal ratings for fearful and happy facial stimuli, and they showed similar recognition rates for previously seen faces (Table 1, Table 2).

\section{BOLD fMRI}

Whole-brain analysis. 
Main effects of Task (across groups). a) Response to valence (fear vs happy): During early presentation (first 40s of face block), fearful compared to happy facial expressions more strongly activated bilateral occipital areas, right middle temporal and angular gyri, and cerebellum. Towards the end of a face block (last 40s), fearful versus happy faces continued to more strongly activate lateral occipital cortices, angular gyri, cerebellum and right occipital fusiform gyrus. In addition, stronger activation was seen in posterior cingulate and precuneus and left temporal fusiform gyrus and hippocampus (Figure 2A, Table S1, Table S2).

b) Response habituation (early vs late): Habituation to fearful faces was associated with decreasing activation in the lateral occipital cortex and left ventrolateral prefrontal cortex (PFC)/ orbitofrontal cortex. Similarly, habituation to happy faces resulted in decreasing activation in occipital areas and left ventrolateral PFC/ orbitofrontal cortex, but also in anterior and posterior cingulate cortices, left hippocampus, amygdala, insula and left temporal and frontal gyri (Figure 2A, Table S1, Table S2).

Drug Group x Task Interaction. a) Response to valence (fear vs happy): During early presentation (first 40s), results identified a significant group $\mathrm{x}$ task interaction in a cluster in the right putamen/ insula (585 voxels, MNI 28,10,-8, $Z=3.63, p=0.04$ ). Post-hoc analyses on BOLD signal change extracted from this cluster indicated that the losartan group showed significantly larger activation in this cluster in response to fearful compared to happy faces ( $t=2.98, d f=14, p=0.010)$. A statistically non-significant trend for an opposite direction of effects in response to fearful versus happy faces was found in the placebo group $(t=1.82$, $d f=14, p=0.090$ ) (between-group t-tests happy/ fear both $t<1.86$, both $d f=28$, both $p>0.073$ ). During the later stage of facial presentation (last 40s), we found a significant group $\mathrm{x}$ task interaction in a cluster in the left middle temporal gyrus (682 voxels, MNI -68,-40,8, Z=4.15, $p=0.03$ ). Post-hoc analyses on BOLD signal change extracted from this cluster indicated that the placebo group showed significantly less activation in this cluster in response to happy (versus fearful) faces in the left middle temporal gyrus $(t=2.52, d f=14, p=0.025)$. No such 
reduction in activation was found in the losartan group ( $t=1.25, d f=14, p=0.233$; betweengroup t-tests: fear $t=0.36, d f=28, p=0.723$, happy $t=2.48, d f=28, p=0.019$ ) (Figure 2B; Table S3).

b) Response habituation (early vs late): There were no group differences in habituation to fearful faces. When comparing early and late BOLD response to happy faces, results showed significant group x task interactions in six clusters, including left putamen/ amygdala (1063 voxels, MNI -28,-12,-4, $Z=3.83, p=0.004)$, right insula (5184 voxels, MNI 36,6,-10, $Z=4.29, p<0.001$ ), anterior cingulate cortex (2196 voxels, MNI -10,4,34, $Z=3.86$, $p<0.001)$, right dorsolateral PFC (1802 voxels, MNI 36,-2,40, $Z=4.69, p<0.001)$, left occipital fusiform (3524 voxels, MNI -38,-62,-18, Z=4.20, $p<0.001$ ) and right angular gyri (1953 voxels, MNI 46,-40,40, $Z=4.27, p<0.001)$. Post-hoc analyses on BOLD signal change extracted from each of these clusters suggested habituation to happy faces in the placebo group, reflected in significant reduction in activation over time in these areas (all $t>2.42$, all $d f=14$, all $p<0.030$ ). No change in response to happy faces was seen in the losartan group (all $t<1.60$, all $d f=14$, all $p>0.132$ ). This effect might have been driven by the drug group already showing lower activation in these areas at the beginning of face presentation, even though independent t-tests only reached statistical significance for the putamen/ amygdala cluster ( $t=2.07, d f=28, p=0.048)$, and non-significant trend-effects for the insula $(p=0.064)$, the occipital fusiform $(p=0.056)$, and the angular gyrus $(p=0.095$; all other $p>0.157$ ) (Figure 2B, Table S3).

\section{ROI analysis.}

Drug Group x Task Interaction. a) Response to valence (fear vs happy): The valence (fear, happy) x time (early, late) x group ANOVA for extracted right amygdala signals revealed a significant valence $\mathrm{x}$ group $(F=4.38, d f=1 / 28, p=0.046)$ but no significant valence $\mathrm{x}$ time $\mathrm{x}$ group effect $(F=0.68, d f=1 / 28, p=0.416)$. Exploring these effects further using 
paired-samples t-tests for fear and happy blocks collapsed across the two time conditions suggested that overall, the losartan group showed lower amygdala response to happy compared to fearful faces ( $t=2.07, d f=14, p=0.050)$, while the placebo group showed similarly high amygdala effects to both valences ( $t=0.76, d f=14, p=0.463$ ) (between-group independent t-tests: fear $t=1.64, d f=28, p=0.112$, happy $t=0.24, d f=28, p=0.816)$. For the left amygdala, similar patterns of activation were observed, even though interaction effects failed to reach statistical significance (all $F<4.12$, all $d f=1 / 28$, all $p>0.052$ ) (Figure 3).

b) Response habituation (early vs late): The valence (fear, happy) x time (early, late) x group ANOVA for right amygdala signal revealed a significant time x group $(F=10.61$, $d f=1 / 28, p=0.003)$ but no significant valence $\mathrm{x}$ time $\mathrm{x}$ group effect $(F=0.68, d f=1 / 28$, $p=0.416)$. Exploring these effects further using paired-samples t-tests for early and late presentations collapsed across the two facial expressions revealed that while the placebo group showed significant habituation effects in response to emotional faces overall $(t=4.38$, $d f=14, p=0.001$ ), amygdala activation remained sustained over time in the losartan group ( $t=0.38, d f=14, p=0.710)$. In line with these observations, amygdala activation was significantly lower in the placebo versus losartan group at the end of face blocks (early: $t=0.70, d f=28, p=0.492$, late: $t=2.18, d f=28, p=0.038)$. For the left amygdala, similar patterns of activation were observed, even though interaction effects failed to reach statistical significance (all $F<4.05$, all $d f=1 / 28$, all $p>0.054$ ) (Figure 3).

Control analysis of hemodynamic response. We also ran an ROI analysis in an anatomical mask of the right occipital fusiform gyrus, a region strongly implicated in face processing (43), as a measure of overall hemodynamic impact of the drug. BOLD \% signal change in response to faces versus baseline was not different between groups (placebo $M=1.10 / S D=0.77 ;$ losartan $M=0.99 / S D=0.61 ; t=0.44 d f=28, p=0.666)$, suggesting that the 
emotion-specific effects of losartan reported above are unlikely to merely be due to the drug leading to global hemodynamic response increases.

\section{Discussion}

Our findings suggest two differential effects of losartan on emotional processing, including i) an improvement of early discrimination of stimuli as threatening versus safe, and ii) a facilitation of threat processing, both processes known to be relevant for exposure to be successful in reducing anxiety $(21,23)$.

\section{Improving Threat-Safety Differentiation}

While during early presentation the placebo group showed similarly high right amygdala activation in response to fearful and happy faces which then decreased over time, the losartan group showed significantly reduced amygdala response to happy faces from the start of presentation. In animal work, losartan has been shown to predominantly target amygdala function, by reducing mRNA levels of AT1 receptors (14). This area is thought to be crucial in threat processing, particularly responding to stimuli associated with danger (44), suggesting that losartan might help to differentiate between threat versus safety signals more rapidly. Furthermore, our results show that losartan mitigated the decrease in middle temporal lobe response to happy faces towards the end of face presentation seen in the placebo group. A recent meta-analysis aiming to identify brain areas associated with fear conditioning (45) identified higher activation in the middle temporal lobe in response to safe compared to threat stimuli, implicating this area as potentially relevant in processing safety signals as well.

It is assumed that a key mechanism of treatment action is inhibitory learning, where the previous association of a stimulus with 'threat' is overwritten by its association with 'safety' (21-23). Successful exposure will result in the individual being able to rapidly discriminate between threat and safety cues, and to suppress fear responses when safety cues 
are present. Strikingly, laboratory research has shown that anxious individuals show deficits in inhibitory learning, reflected in stronger response to threat and safety stimuli during conditioning and fear extinction (46-49), and in decreased response in brain areas associated with successful extinction, such as the orbitofrontal and medial prefrontal cortex (50-53). Such findings suggest that successful exposure depends on strong inhibitory learning, and that individuals with anxiety disorders are in need of strategies to enhance such processes (21).

As a complimentary approach, it might be possible to compensate for deficits in inhibitory learning and threat-safety discrimination seen in anxious patients prior to exposure by using pharmacological add-on compounds such as losartan. Even though this remains to be demonstrated more directly in fear learning and extinction paradigms, our results suggest that the drug might improve rapid discrimination of safety cues.

\section{Improving Threat Processing during Exposure}

Our findings also suggest that losartan increases brain response to fearful faces in areas associated with threat processing. First, while the placebo group showed signal decline in the right amygdala over time, the initial level of amygdala response remained sustained over time in the losartan group, suggesting sustained processing of threat stimuli. Secondly, while the placebo group showed similar levels of response in the right putamen and insula to fearful and happy faces during early presentation, response to fearful faces was significantly increased after losartan administration.

Amygdala and insula are crucial parts of the fear circuit of the brain, with the amygdala predominantly being involved in threat detection and processing (54), and the insula being implicated in awareness of internal physiological and emotional states (55-57). Increased activation in these areas can be seen during extinction learning and recall (58-61). Similarly, putamen response has been associated with enhanced threat processing (62-64), 
and reduced activity has been seen to correlate with directing attention away from threat stimuli as a form of avoidance (65).

Avoidance of threat stimuli - attentional, cognitive-emotional, or physical - is thought to play a crucial role in maintaining anxiety, as it prevents the patient from experiencing the absence of the anticipated catastrophe. Hence, for successful inhibitory learning to take place during exposure, it is essential for attentional and emotional processing to be focused on the threat stimulus for a prolonged period of time, to allow prediction error correction (21-23). Accordingly, experimental procedures guiding attention towards threat stimuli during exposure have been demonstrated to have facilitative effects on exposure outcome, while manipulations interfering with threat processing, such as cognitive load or safety behaviours, severely reduce the clinical effects of exposure $(66,67)$. In line with this argumentation, experimental research suggests that anxiety patients who show particularly pronounced attentional avoidance for threat prior to exposure therapy benefited less from treatment than patients who showed prolonged attention towards threat stimuli $(68,69)$. Neuroimaging research also shows that increased activation in brain areas previously associated with threat processing (4), such as the amygdala and insula, before exposure- predicts improved clinical response (70-74), further strengthening the argument that better treatment outcome might depend on increased responsiveness in brain areas involved in threat processing.

We propose that the effects of losartan seen in this study, an increased or sustained activation in amygdala, putamen and insula in response to fearful faces, provide an additional potential pathway by which the drug might augment exposure in humans, where losartan enhances threat processing prior to psychological treatment.

While these results are promising, there are limitations to their interpretation. First, even though our results provide evidence that losartan alters differentiation between fearful and happy faces, follow-up research using fear conditioning designs where threat versus 
safety associations of originally neutral stimuli are learned are needed to confirm that the changes in processing highly complex, salient facial stimuli seen here translate to experimental designs more representative of fear acquisition and extinction. Secondly, one might wonder whether interpreting the increase in amygdala response to threat following losartan as potentially promising for augmenting exposure therapy is justified, considering that activation in this area is characteristically increased in anxiety. However, an anxiogenic interpretation of the losartan effects would not explain the reduction of amygdala response to happy faces following losartan, an effect that suggests improved safety learning, which is usually impaired in anxiety disorders (46-49). In line with our interpretation of losartan action, research shows that an increased pre-treatment amygdala response to negative stimuli predicts improved clinical outcome after exposure therapy (70-74), suggesting that losartan might improve treatment effects by boosting processing in this area prior to CBT. Nevertheless, the actual effect of losartan on exposure therapy in clinical samples remains to be investigated directly, to be able to fully evaluate the drug's potential in improving clinical outcome. Thirdly, we propose in our interpretation of results that losartan prevented habituation in amygdala response to fearful but not happy faces, based on significant valence $\mathrm{x}$ group and time $\mathrm{x}$ group effects. Even though our interpretation is valid, assuming that after losartan amygdala activation remained high in response to fearful faces over time while remaining low in response to happy faces, a significant three-way interaction would provide final evidence for this assumption. Furthermore, the effects of losartan on emotion processing observed here should be replicated in larger samples.

Taken together, this study has identified two potential mechanisms by which losartan might benefit clinical outcome of exposure for anxiety disorders. The results provide evidence that a single dose of losartan, in the absence of overall effects on blood pressure, heart rate, or mood, amplifies activation in brain areas relevant to emotional processing when processing 
threat stimuli, while directing such resources away from non-threat stimuli. We propose that these effects might positively interact with exposure and improve clinical outcome, by facilitating safety learning, and by augmenting thorough threat processing and predictionerror correction during exposure. Such knowledge will ultimately help to optimally combine this and similar pharmacological agents with psychological treatment, and it will drive the development of more accessible, more compact treatment formats targeting the mechanisms of CBT action more effectively.

\section{Funding, disclosure, acknowledgements}

This research was funded by a MQ: Transforming Mental Health fellowship awarded to AR.

AR, JKB, NK and MGC report no conflicts of interest. MB has received travel expenses from Lundbeck for attending conferences. KJR is on the Scientific Advisory Boards for Resilience Therapeutics, Sheppard Pratt-Lieber Research Institute, Laureate Institute for Brain Research, The Army STARRS Project, UCSD VA Center of Excellence for Stress and Mental Health - CESAMH, and the Anxiety and Depression Association of America. He provides fee-for-service consultation for Biogen and Resilience Therapeutics. He holds patents for use of d-cycloserine and psychotherapy, targeting PAC1 receptor for extinction, targeting tachykinin 2 for prevention of fear, targeting angiotensin to improve extinction of fear. KJR is also founding member of Extinction Pharmaceuticals to develop d-cycloserine to augment the effectiveness of psychotherapy, for which he has received no equity or income within the last 3 years. CJH has received consultancy fees from Lundbeck, Servier, Johnson and Johnson and P1vital. 


\section{References}

1. Bontempo A, Panza KE, Bloch MH. D-Cycloserine Augmentation of Behavioral Therapy for the Treatment of Anxiety Disorders: A Meta-Analysis. Journal of Clinical Psychiatry. 2012;73(4):533-7.

2. Norberg MM, Krystal JH, Tolin DF. A meta-analysis of D-Cycloserine and the facilitation of fear extinction and exposure therapy. Biological psychiatry. 2008;63(12):111826.

3. Xia J, Du Y, Han J, Liu G, Wang X. D-cycloserine augmentation in behavioral therapy for obsessive-compulsive disorder: a meta-analysis. Drug design, development and therapy. 2015;9:2101-17.

4. Reinecke A, Harmer C. A cognitive-neuropsychological account of treatment action in anxiety: can we augment clinical efficacy? Psychopathology Review. 2015;3(1):77-109.

5. Mataix-Cols D, de la Cruz LF, Monzani B, Rosenfield D, Andersson E, Perez-Vigil A, et al. D-Cycloserine Augmentation of Exposure-Based Cognitive Behavior Therapy for Anxiety, Obsessive-Compulsive, and Posttraumatic Stress Disorders A Systematic Review and Meta-analysis of Individual Participant Data. Jama Psychiatry. 2017;74(5):501-10. 6. Fogari R, Mugellini A, Zoppi A, Derosa G, Pasotti C, Fogari E, et al. Influence of losartan and atenolol on memory function in very elderly hypertensive patients. Journal of human hypertension. 2003;17(11):781-5.

7. Mechaeil R, Gard P, Jackson A, Rusted J. Cognitive enhancement following acute losartan in normotensive young adults. Psychopharmacology. 2011;217(1):51-60.

8. Tedesco MA, Ratti G, Mennella S, Manzo G, Grieco M, Rainone AC, et al. Comparison of losartan and hydrochlorothiazide on cognitive function and quality of life in hypertensive patients. American journal of hypertension. 1999;12(11 Pt 1):1130-4.

9. Khachaturian AS, Zandi PP, Lyketsos CG, Hayden KM, Skoog I, Norton MC, et al. Antihypertensive medication use and incident Alzheimer disease: the Cache County Study. Archives of neurology. 2006;63(5):686-92.

10. Mogi M, Horiuchi M. Effects of angiotensin II receptor blockers on dementia. Hypertension research : official journal of the Japanese Society of Hypertension. 2009;32(9):738-40.

11. Khoury NM, Marvar PJ, Gillespie CF, Wingo A, Schwartz A, Bradley B, et al. The renin-angiotensin pathway in posttraumatic stress disorder: angiotensin-converting enzyme inhibitors and angiotensin receptor blockers are associated with fewer traumatic stress symptoms. The Journal of clinical psychiatry. 2012;73(6):849-55.

12. Lazaroni TLD, Bastos CP, Moraes MFD, Santos RS, Pereira GS. Angiotensin-(17)/Mas axis modulates fear memory and extinction in mice. Neurobiology of learning and memory. 2016;127:27-33.

13. Quinones MM, Maldonado L, Velazquez B, Porter JT. Candesartan ameliorates impaired fear extinction induced by innate immune activation. Brain Behavior and Immunity. 2016;52:169-77.

14. Marvar PJ, Goodman J, Fuchs S, Choi DC, Banerjee S, Ressler KJ. Angiotensin type 1 receptor inhibition enhances the extinction of fear memory. Biological psychiatry. 2014;75(11):864-72.

15. Wright JW, Harding JW. The brain renin-angiotensin system: a diversity of functions and implications for CNS diseases. Pflugers Archiv : European journal of physiology. 2013;465(1):133-51.

16. Chai SY, Bastias MA, Clune EF, Matsacos DJ, Mustafa T, Lee JH, et al. Distribution of angiotensin IV binding sites (AT4 receptor) in the human forebrain, midbrain and pons as visualised by in vitro receptor autoradiography. Journal of chemical neuroanatomy.

2000;20(3-4):339-48. 
17. Harding JW, Cook VI, Miller-Wing AV, Hanesworth JM, Sardinia MF, Hall KL, et al. Identification of an AII(3-8) [AIV] binding site in guinea pig hippocampus. Brain research. 1992;583(1-2):340-3.

18. Armando I, Carranza A, Nishimura Y, Hoe KL, Barontini M, Terron JA, et al. Peripheral administration of an angiotensin II AT(1) receptor antagonist decreases the hypothalamic-pituitary-adrenal response to isolation Stress. Endocrinology. 2001;142(9):3880-9.

19. Davis M, Walker DL, Lee Y. Amygdala and bed nucleus of the stria terminalis: differential roles in fear and anxiety measured with the acoustic startle reflex. Philosophical transactions of the Royal Society of London Series B, Biological sciences. 1997;352(1362):1675-87.

20. Hurt RC, Garrett JC, Keifer OP, Linares A, Couling L, Speth RC, et al. Angiotensin type $1 \mathrm{a}$ receptors on corticotropin-releasing factor neurons contribute to the expression of conditioned fear(1). Genes Brain and Behavior. 2015;14(7):526-33.

21. Craske MG, Treanor M, Conway CC, Zbozinek T, Vervliet B. Maximizing exposure therapy: an inhibitory learning approach. Behaviour research and therapy. 2014;58:10-23.

22. Craske MG, Kircanski K, Zelikowsky M, Mystkowski J, Chowdhury N, Baker A. Optimizing inhibitory learning during exposure therapy. Behaviour research and therapy. 2008;46(1):5-27.

23. Milad MR, Rosenbaum BL, Simon NM. Neuroscience of fear extinction: implications for assessment and treatment of fear-based and anxiety related disorders. Behaviour research and therapy. 2014;62:17-23.

24. Britton JC, Gold AL, Feczko EJ, Rauch SL, Williams D, Wright CI. D-cycloserine inhibits amygdala responses during repeated presentations of faces. CNS spectrums. 2007;12(8):600-5.

25. Wright CI, Fischer H, Whalen PJ, McInerney SC, Shin LM, Rauch SL. Differential prefrontal cortex and amygdala habituation to repeatedly presented emotional stimuli. Neuroreport. 2001;12(2):379-83.

26. Spielberger CD, Gorsuch, R.L., Lushene, R., Vagg, P.R., \& Jacobs, G.A. Manual for State-Trait Anxiety Inventory. Palo Alto, CA: Consulting Psychologists Press; 1983.

27. Bishop SJ, Jenkins R, Lawrence AD. Neural processing of fearful faces: effects of anxiety are gated by perceptual capacity limitations. Cerebral cortex (New York, NY : 1991). 2007;17(7):1595-603.

28. Fox E, Yates A, Ashwin C. Trait anxiety and perceptual load as determinants of emotion processing in a fear conditioning paradigm. Emotion (Washington, DC). 2012;12(2):236-49.

29. First MB, Spitzer RL, Gibbon M, Williams JBW. Structured clinical interview for DSM-IV axis I disorders, Clinician Version (SCID-CV). Washington, DC: American Psychiatry Press, Inc.; 1996.

30. Taylor S, Cox BJ. An expanded anxiety sensitivity index: evidence for a hierarchic structure in a clinical sample. J Anxiety Disord. 1998;12(5):463-83.

31. Eysenck HJ, Eysenck SBG. Manual of the Eysenck Personality Questionnaire (Junior and Adult). Kent, UK: Hodder \& Stoughton; 1975.

32. Carver CS, White TL. Behavioral inhibition, behavioral activation, and affective responses to impending reward and punishment - the BIS BAS Scales. Journal of Personality and Social Psychology. 1994;67(2):319-33.

33. Beck AT, Steer RA, Brown GK. Manual for the Beck Depression Inventory-II. San Antonio, TX: Psychological Corporation; 1996.

34. Derryberry D, Reed MA. Anxiety-related attentional biases and their regulation by attentional control. Journal of abnormal psychology. 2002;111(2):225-36. 
35. Nelson HE. National adult reading test (NART): Tets manual. Windsor: NFERNelson; 1982.

36. Lo MW, Goldberg MR, McCrea JB, Lu H, Furtek CI, Bjornsson TD.

Pharmacokinetics of losartan, an angiotensin II receptor antagonist, and its active metabolite EXP3174 in humans. Clinical pharmacology and therapeutics. 1995;58(6):641-9.

37. Ohtawa M, Takayama F, Saitoh K, Yoshinaga T, Nakashima M. Pharmacokinetics and biochemical efficacy after single and multiple oral administration of losartan, an orally active nonpeptide angiotensin II receptor antagonist, in humans. British journal of clinical pharmacology. 1993;35(3):290-7.

38. Tottenham N, Tanaka JW, Leon AC, McCarry T, Nurse M, Hare TA, et al. The NimStim set of facial expressions: Judgments from untrained research participants. Psychiatry research. 2009;168(3):242-9.

39. Jenkinson M, Bannister P, Brady M, Smith S. Improved optimization for the robust and accurate linear registration and motion correction of brain images. Neuroimage. 2002;17(2):825-41.

40. Smith SM. Fast robust automated brain extraction. Human Brain Mapping. 2002;17(3):143-55.

41. Woolrich MW, Ripley BD, Brady M, Smith SM. Temporal autocorrelation in univariate linear modeling of FMRI data. Neuroimage. 2001;14(6):1370-86.

42. Beckmann CF, Jenkinson M, Smith SM. General multilevel linear modeling for group analysis in FMRI. Neuroimage. 2003;20(2):1052-63.

43. Kanwisher N, McDermott J, Chun MM. The fusiform face area: a module in human extrastriate cortex specialized for face perception. The Journal of neuroscience : the official journal of the Society for Neuroscience. 1997;17(11):4302-11.

44. Etkin A. Functional Neuroanatomy of Anxiety: A Neural Circuit Perspective. In: Stein MB, Steckler T, editors. Behavioral Neurobiology of Anxiety and Its Treatment. 22010. p. 251-77.

45. Fullana MA, Harrison BJ, Soriano-Mas C, Vervliet B, Cardoner N, Avila-Parcet A, et al. Neural signatures of human fear conditioning: an updated and extended meta-analysis of fMRI studies. Molecular psychiatry. 2016;21(4):500-8.

46. Blechert J, Michael T, Vriends N, Margraf J, Wilhelm FH. Fear conditioning in posttraumatic stress disorder: evidence for delayed extinction of autonomic, experiential, and behavioural responses. Behaviour research and therapy. 2007;45(9):2019-33.

47. Craske MG, Waters AM, Lindsey Bergman R, Naliboff B, Lipp OV, Negoro H, et al. Is aversive learning a marker of risk for anxiety disorders in children? Behaviour research and therapy. 2008;46(8):954-67.

48. Lissek S, Powers AS, McClure EB, Phelps EA, Woldehawariat G, Grillon C, et al. Classical fear conditioning in the anxiety disorders: a meta-analysis. Behaviour research and therapy. 2005;43(11):1391-424.

49. Milad MR, Pitman RK, Ellis CB, Gold AL, Shin LM, Lasko NB, et al. Neurobiological basis of failure to recall extinction memory in posttraumatic stress disorder. Biological psychiatry. 2009;66(12):1075-82.

50. Indovina I, Robbins TW, Nunez-Elizalde AO, Dunn BD, Bishop SJ. Fear-conditioning mechanisms associated with trait vulnerability to anxiety in humans. Neuron. 2011;69(3):56371.

51. Jovanovic T, Norrholm SD. Neural mechanisms of impaired fear inhibition in posttraumatic stress disorder. Frontiers in behavioral neuroscience. 2011;5:44.

52. Milad MR, Furtak SC, Greenberg JL, Keshaviah A, Im JJ, Falkenstein MJ, et al. Deficits in conditioned fear extinction in obsessive-compulsive disorder and neurobiological changes in the fear circuit. JAMA Psychiatry. 2013;70(6):608-18; quiz 554. 
53. Rougemont-Bucking A, Linnman C, Zeffiro TA, Zeidan MA, Lebron-Milad K, Rodriguez-Romaguera J, et al. Altered processing of contextual information during fear extinction in PTSD: an fMRI study. CNS neuroscience \& therapeutics. 2011;17(4):227-36. 54. Shin LM, Liberzon I. The neurocircuitry of fear, stress, and anxiety disorders. Neuropsychopharmacology : official publication of the American College of Neuropsychopharmacology. 2010;35(1):169-91.

55. Craig AD. How do you feel - now? The anterior insula and human awareness. Nature Reviews Neuroscience. 2009;10(1):59-70.

56. Critchley HD, Wiens S, Rotshtein P, Ohman A, Dolan RJ. Neural systems supporting interoceptive awareness. Nature Neuroscience. 2004;7(2):189-95.

57. Zaki J, Davis JI, Ochsner KN. Overlapping activity in anterior insula during interoception and emotional experience. Neuroimage. 2012;62(1):493-9.

58. Gottfried JA, Dolan RJ. Human orbitofrontal cortex mediates extinction learning while accessing conditioned representations of value. Nat Neurosci. 2004;7(10):1144-52.

59. LaBar KS, Gatenby JC, Gore JC, LeDoux JE, Phelps EA. Human amygdala activation during conditioned fear acquisition and extinction: a mixed-trial fMRI study. Neuron. 1998;20(5):937-45.

60. Milad MR, Wright CI, Orr SP, Pitman RK, Quirk GJ, Rauch SL. Recall of fear extinction in humans activates the ventromedial prefrontal cortex and hippocampus in concert. Biological psychiatry. 2007;62(5):446-54.

61. Phelps EA, Delgado MR, Nearing KI, LeDoux JE. Extinction learning in humans: Role of the amygdala and vmPFC. Neuron. 2004;43(6):897-905.

62. Hasler G, Fromm S, Alvarez RP, Luckenbaugh DA, Drevets WC, Grillon C. Cerebral blood flow in immediate and sustained anxiety. The Journal of neuroscience : the official journal of the Society for Neuroscience. 2007;27(23):6313-9.

63. Preibisch C, Lanfermann H, Wallenhorst T, Walter H, Erk S. Neuroanatomical correlates of visual field bias: a sensitive system for detecting potential threats? Brain research. 2009;1263:69-77.

64. Uono S, Sato W, Kochiyama T, Kubota Y, Sawada R, Yoshimura S, et al. Putamen Volume is Negatively Correlated with the Ability to Recognize Fearful Facial Expressions. Brain topography. 2017;30(6):774-84.

65. Maresh EL, Beckes L, Coan JA. The social regulation of threat-related attentional disengagement in highly anxious individuals. Frontiers in human neuroscience. 2013;7:515. 66. Mulken S, Bogels SM, de Jong PJ, Louwers J. Fear of blushing: effects of task concentration training versus exposure in vivo on fear and physiology. J Anxiety Disord. 2001;15(5):413-32.

67. Sloan T, Telch MJ. The effects of safety-seeking behavior and guided threat reappraisal on fear reduction during exposure: an experimental investigation. Behaviour research and therapy. 2002;40(3):235-51.

68. Barry TJ, Sewart AR, Arch JJ, Craske MG. Deficits in disengaging attention from threat predict improved response to cognitive behavioral therapy for anxiety. Depress Anxiety. 2015;32(12):892-9.

69. Price M, Tone EB, Anderson PL. Vigilant and avoidant attention biases as predictors of response to cognitive behavioral therapy for social phobia. Depress Anxiety. 2011;28(4):349-53.

70. McClure EB, Adler A, Monk CS, Cameron J, Smith S, Nelson EE, et al. fMRI predictors of treatment outcome in pediatric anxiety disorders. Psychopharmacology. 2007;191(1):97-105.

71. Olatunji BO, Ferreira-Garcia R, Caseras X, Fullana MA, Wooderson S, Speckens A, et al. Predicting response to cognitive behavioral therapy in contamination-based obsessive- 
compulsive disorder from functional magnetic resonance imaging. Psychological medicine. 2013;12:1-13.

72. Doehrmann O, Ghosh SS, Polli FE, Reynolds GO, Horn F, Keshavan A, et al. Predicting Treatment Response in Social Anxiety Disorder From Functional Magnetic Resonance Imaging. Jama Psychiatry. 2013;70(1):87-97.

73. Klumpp H, Fitzgerald DA, Phan KL. Neural predictors and mechanisms of cognitive behavioral therapy on threat processing in social anxiety disorder. Progress in neuropsychopharmacology \& biological psychiatry. 2013;45:83-91.

74. Reinecke A, Thilo K, Filippini N, Croft A, Harmer CJ. Predicting rapid response to cognitive-behavioural treatment for panic disorder: The role of hippocampus, insula, and dorsolateral prefrontal cortex. Behaviour research and therapy. 2014;62:120-8. 
Table 1: Sociodemographic, clinical and personality characteristics, physiological measures and experimental stimuli ratings of high-trait anxious participants in the losartan versus placebo group ( $M, S D$, and $t$-test/ $X^{2}$-test $p$-scores).

\begin{tabular}{|c|c|c|c|c|c|}
\hline & \multicolumn{2}{|c|}{ Losartan $(N=15)$} & \multicolumn{2}{|c|}{ Placebo $(N=15)$} & \multirow[b]{2}{*}{$P$} \\
\hline & $M$ & $S D$ & $M$ & $S D$ & \\
\hline \multicolumn{6}{|l|}{ Sociodemographic Data } \\
\hline Gender & \multicolumn{2}{|c|}{11 \%/ 4 ठ } & \multicolumn{2}{|c|}{$11 q / 4 \widehat{0}$} & \\
\hline Age & 22.4 & 3.9 & 22.4 & 4.8 & \\
\hline Verbal IQ (NART) & 114.1 & 6.2 & 115.9 & 6.9 & \\
\hline \multicolumn{6}{|l|}{ Clinical and Personality Measures } \\
\hline Trait Anxiety (STAIT) & 53.3 & 7.3 & 51.5 & 6.1 & \\
\hline Anxiety Sensitivity (ASI) & 29.0 & 15.9 & 33.4 & 24.6 & \\
\hline Behavioral Activation (BAS) & 24.6 & 4.9 & 23.2 & 4.2 & \\
\hline Behavioral Inhibition (BIS) & 13.6 & 3.3 & 12.9 & 2.7 & \\
\hline Beck Depression Inventory (BDI) & 8.9 & 5.7 & 11.4 & 8.4 & \\
\hline Neuroticism (EPQ) & 14.9 & 6.5 & 12.8 & 5.6 & \\
\hline \multicolumn{6}{|l|}{ Attentional Control (ACS) } \\
\hline Total & 53.0 & 7.4 & 51.3 & 7.0 & \\
\hline Focusing & 23.0 & 3.8 & 21.9 & 4.5 & \\
\hline Shifting & 30.0 & 4.7 & 29.4 & 5.1 & \\
\hline \multicolumn{6}{|l|}{ Physiological Measures } \\
\hline Heart rate - Baseline & 81.9 & 14.6 & 83.3 & 11.6 & \\
\hline Heart rate - Drug Peak & 73.1 & 8.7 & 70.4 & 10.4 & .46 \\
\hline Systolic blood pressure - Baseline & 115.4 & 14.3 & 120.7 & 16.9 & \\
\hline Systolic blood pressure - Drug Peak & 116.6 & 12.5 & 114.7 & 14.8 & .72 \\
\hline Diastolic blood pressure - Baseline & 73.1 & 11.3 & 73.8 & 9.5 & \\
\hline Diastolic blood pressure - Drug Peak & 71.2 & 6.9 & 70.2 & 9.0 & .74 \\
\hline \multicolumn{6}{|l|}{ Experimental Scan Measures } \\
\hline Recognition accuracy - fear & 0.53 & 0.40 & 0.77 & 0.26 & .07 \\
\hline Recognition accuracy - happy & 0.50 & 0.42 & 0.60 & 0.39 & .51 \\
\hline Valence rating - fear & -1.2 & 1.4 & -1.7 & 0.8 & .24 \\
\hline Valence rating - happy & 1.3 & 1.4 & 2.0 & 0.7 & .10 \\
\hline Arousal rating - fear & 3.7 & 1.0 & 3.7 & 1.0 & .90 \\
\hline Arousal rating - happy & 3.2 & 1.3 & 2.9 & 1.1 & .56 \\
\hline
\end{tabular}

Note: NART = National Adult Reading Test; STAIT = State-Trait Anxiety Inventory; ASI = Anxiety Sensitivity Index; BAS = Behavioral Activation Scale; BIS = Behavioral Inhibition Scale; BDI = Beck Depression Inventory; EPQ = Eysenck Personality Inventory; ACS = Attentional Control Scale. 
Table 2. Visual analogue scale ratings in the two groups before drug intake and at drug peak-level ( $M, S D$, and $t$-test $p$-scores).

\begin{tabular}{|c|c|c|c|c|c|c|c|c|c|}
\hline & \multicolumn{4}{|c|}{ Baseline } & \multicolumn{4}{|c|}{ Drug Peak Level } & \multirow[b]{3}{*}{$P$} \\
\hline & \multicolumn{2}{|c|}{ Losartan } & \multicolumn{2}{|c|}{ Placebo } & \multicolumn{2}{|c|}{ Losartan } & \multicolumn{2}{|c|}{ Placebo } & \\
\hline & $M$ & $S D$ & $M$ & $S D$ & $M$ & $S D$ & $M$ & $S D$ & \\
\hline \multicolumn{10}{|c|}{ Visual Analogue Ratings VAS } \\
\hline Anxious & 28 & 23 & 26 & 20 & 16 & 19 & 23 & 21 & .40 \\
\hline Tearful & 12 & 20 & 10 & 16 & 5 & 10 & 5 & 9 & .94 \\
\hline Hopeless & 11 & 20 & 13 & 18 & 6 & 14 & 12 & 20 & .36 \\
\hline Sad & 11 & 17 & 15 & 22 & 7 & 10 & 11 & 17 & .43 \\
\hline Depressed & 12 & 17 & 12 & 16 & 5 & 8 & 9 & 13 & .34 \\
\hline Sleepy & 27 & 26 & 21 & 17 & 23 & 24 & 19 & 11 & .59 \\
\hline Nauseous & 11 & 18 & 3 & 7 & 7 & 12 & 2 & 4 & .14 \\
\hline Dizzy & 6 & 12 & 5 & 7 & 4 & 6 & 7 & 17 & .58 \\
\hline Heart racing & 15 & 19 & 12 & 15 & 11 & 16 & 5 & 5 & .15 \\
\hline Alert & 54 & 21 & 46 & 27 & 48 & 25 & 38 & 25 & .27 \\
\hline
\end{tabular}


Figure 1. Experimental task procedure. Each participant was presented with four face blocks, including two happy blocks and two fear blocks, each showing a different person. Half of the participants in each group started with a happy block, and half of the participants started with a fear block.

Figure 2. A. Whole-brain fMRI main effect of task: Response to valence: Across groups, fearful versus happy facial expressions predominantly activated bilateral occipital areas during early presentation, and bilateral occipital areas, posterior cingulate, precuneus and left hippocampus during late presentation. Response habituation: Across groups, habituation to emotional faces was associated with decreasing activation in bilateral occipital areas and left ventrolateral PFC/ OFC. Habituation to happy faces also resulted in a decrease in anterior and posterior cingulate, and left insula, hippocampus and amygdala. B. Whole-brain fMRI task x group interaction. Response to valence: Early during face presentation, losartan lead to a significant increase in activation in the right putamen/ insula in response to fearful versus happy faces (i). Towards the end of face presentation, losartan sustained similar levels of activation in the left middle temporal gyrus in response to happy versus fearful faces, while placebo was associated with a significant decrease in activation in this area towards happy faces. Response habituation: The placebo group but not the losartan group showed a significant decrease in activation over time in response to happy faces in left putamen/ amygdala, right insula, anterior cingulate, right dorsolateral PFC, left occipital fusiform gyrus, and right angular gyrus.

Figure 3. Region-of-interest analysis in the anatomical left and right amygdala. Group interactions were not significant for the left amygdala. For the right amygdala, the placebo group showed similarly high amygdala signal in response to both emotions, but the losartan group showed significantly lower amygdala response to happy compared to fearful faces. 
Also, the placebo group showed significant reduction of activation over time in both valence conditions, suggesting habituation effects. In contrast, amygdala activation remained sustained over time in the losartan group. Error bars show s.e.m. 